\title{
Relationship between Micro-Incompatibility and Heterogeneity of Dislocation Density Distribution in Cu-9at.\% Al Symmetric Type Bicrystal Models under Tensile Loading
}

\author{
Ryouji KONDOU $^{* *}$, Tetsuya OHASHI ${ }^{* * *}$ and Sei MIURA ${ }^{* * * *}$ \\ ${ }^{* *}$ Faculty of Engineering, University of the Ryukyus, \\ 1 Senbaru, Nishihara-cho, Nakagami-gun, Okinawa 903-0213, Japan \\ E-mail: kondou@teada.tec.u-ryukyu.ac.jp \\ ***Kitami Institute of Technology, \\ 165 Koen-cho, Kitami-shi, Hokkaido 090-8507, Japan \\ E-mail: ohashi@newton.mech.kitami-it.ac.jp \\ ****Sojo University, \\ 4-22-1 Ikeda, Kumamoto 860-0082, Japan \\ E-mail: sei-miura@mb.0038.net
}

\begin{abstract}
Slip deformation in Cu-9at.\% Al symmetric type bicrystal models subjected to tensile loading is investigated by a finite element crystal plasticity analysis code. Accumulation of geometrically necessary dislocations (GNDs) and statistically stored dislocations (SSDs) are studied in detail. Results of the analysis show asymmetric non-uniform deformation and accumulation of GNDs on the primary and secondary slip systems with activation of secondary slip system near the grain boundary. Mechanism of asymmetric non-uniform deformation with GNDs accumulated near the grain boundary in the $\mathrm{Cu}-9$ at.\% $\mathrm{Al}$ symmetric type bicrystal models is discussed from the viewpoint of the effects of the elastic anisotropy of $\mathrm{Cu}-9 \mathrm{at} . \% \mathrm{Al}$ and the heterogeneity of initial statically stored dislocations density.
\end{abstract}

Key words: Micro-Incompatibility, Cu-9 at.\% Al Symmetric Type Bicrystals, Crystal Plasticity Analysis, Geometrically Necessary Dislocation, Statically Stored Dislocation, Elastic Anisotropy

\section{Introduction}

Since the plastic deformation of polycrystalline metal is caused by a combination of the slip deformation and elastic deformation of each crystal grain, the restraint interaction of deformation occurs through the grain boundary plane. For understanding the effects of the grain boundary, the investigation of bicrystals that is most basic model of polycrystalline models is effective. When the plastic deformation take place in bicrystals, normally, in order to satisfy the continuity of displacement at the grain boundary, non-uniform deformation occurs near the grain boundary due to the effects of the elastic incompatibility stress $^{(1)(2)}$, the strain incompatibility ${ }^{(1)}$ and the stress concentration caused by dislocation accumulation at the grain boundary ${ }^{(3)}$. Since the various effects of the grain boundary are piled up, non-uniform deformation near the grain boundary is complicated problem.

In the case of the dynamical factor complicatedly participate in phenomenon; numerical studies are effective for understanding the relationship between the dynamical factor and result. For example, Ohashi have performed analysis of elastic incompatibility stress in the vicinity of a grain boundary plane, assuming anisotropic bicrystals of three-dimensional geometry and using the finite elemental method, and stated in a previous study ${ }^{(4)}$, slip process strongly depend on elastic stress field in the early stage of deformation.

However, more complicated phenomenon have stated in a previous experimental study. 
For example, Miura have performed observation of deformation of $\mathrm{Cu}-9 \mathrm{at} . \% \mathrm{Al}$ symmetric type bicrystals accompanying tensile loading, and stated in a previous study ${ }^{(5)(6)}$, non-uniform deformation occurs near the grain boundary, dislocation sources are restricted in the local area near the grain boundary, the grain boundary plane dose not work as dislocation source, even if the secondary slip system near the grain boundary is activated, that is, the so-called "micro-incompatibility"(5) (6)

Concerning the scale of characteristic, "micro incompatibility" at the grain boundary is far deferent from microscopic non-uniform slip deformation behavior. In this case, investigation of the microscopic motion and accumulation of dislocation that is cause of the slip deformation behavior is effective for understanding comprehensively.

In this study, to clarify the mechanism of "micro incompatibility" at the grain boundary, crystal plasticity analysis of tensile deformation of Cu-9at.\% Al symmetric type bicrystals was performed, and the slip deformation behavior and distribution of the dislocation density was discussed.

\section{Analysis Method}

\subsection{Analysis of deformation by finite element method}

If it is assumed that the activation condition of the slip system is given by the Schmid law, the following equation will hold between the stress tensor $\sigma_{i j}$ under the activation condition and the critical resolved shear stress $\theta^{(n)}$ in the $n$-th slip system:

$$
\begin{aligned}
& P_{i j}^{(n)} \sigma_{i j}=\theta^{(n)} \\
& P_{i j}^{(n)} \dot{\sigma}_{i j}=\dot{\theta}^{(n)} \\
& P_{i j}^{(n)}=\frac{1}{2}\left\{v_{i}^{(n)} b_{j}^{(n)}+v_{j}^{(n)} b_{i}^{(n)}\right\}
\end{aligned}
$$

where, $P_{i j}^{(n)}$ is the Schmid tensor in the $n$-th slip system, wherein the unit vector in the plane normal direction is $v_{i}^{(n)}$ and the unit vector in the slip direction is $b_{i}^{(n)}$.

The strain increment $\dot{\varepsilon}_{i j}$ is acquired from the sum of the elastic component $\dot{\varepsilon}_{i j}^{e}$ and the plastic component $\dot{\varepsilon}_{i j}^{p}$ using the following equation:

$$
\begin{aligned}
& \dot{\varepsilon}_{i j}=\dot{\varepsilon}_{i j}^{e}+\dot{\varepsilon}_{i j}^{p} \\
& \dot{\varepsilon}_{i j}^{e}=S_{i j k l}^{e} \dot{\sigma}_{k l} \\
& \dot{\varepsilon}_{i j}^{p}=\sum_{n} \dot{\gamma}^{(n)} P_{i j}^{(n)}
\end{aligned}
$$

where, $S_{i j k l}^{e}$ is an elastic compliance and $\dot{\gamma}^{(n)}$ is a plastic shear strain increment.

If it is assumed that the strain hardening coefficient is $h^{(\mathrm{nm})}$ and the strain hardening law is represented by the following equation:

$$
\dot{\theta}^{(n)}=\sum_{m} h^{(n m)} \dot{\gamma}^{(m)}
$$

then, the elastic/plastic constitutive equation ${ }^{(7)}$ is represented by the following equation:

$$
\dot{\sigma}_{i j}=\left[S_{i j k l}^{e}+\sum_{n} \sum_{m}\left\{h^{(n m)}\right\}^{-1} P_{i j}^{(n)} P_{k l}^{(m)}\right]^{-1} \dot{\varepsilon}_{k l}
$$

However, $n$ and $m$ are added to obtain their sum only in active slip systems. Based on the elastic/plastic constitutive equation, the deformation of the model is analyzed using the finite element method.

\section{2:2 Analysis of dislocation density}

Dislocations present inside the material having been subjected to deformation hysteresis are roughly classified into two kinds, that is, "statistically stored dislocations" (SS dislocations) and "geometrically necessary dislocations" (GN dislocations) ${ }^{(8)}$, and their density is calculated from the strain increment, which is the result of analyzing deformation. The relationship between the increment $\dot{\rho}_{S}^{(n)}$ of the SS dislocation density and $\dot{\gamma}^{(n)}$ is 
represented by the following equation ${ }^{(9)}$ :

$$
\dot{\rho}_{S}^{(n)}=\frac{c \dot{\gamma}^{(n)}}{\tilde{b} L^{(n)}}
$$

where, $c$ is a coefficient of the order of $1, \tilde{b}$ is the magnitude of the Burgers vector, and $L^{(n)}$ is an mean free path of dislocation.

The slip deformation of the crystal grain is produced by the motion of dislocations, and therefore, in the area in which dislocations have passed, slip deformation occurs and in the area in which dislocations have not passed yet, no slip deformation occurs. Consequently, at a place where a spatial gradient of $\gamma^{(m)}$ is produced, dislocations must exist. This is called the GN dislocation ${ }^{(8)}$.

If it is assumed that the direction parallel to the slip direction on the slip plane is $\xi^{(m)}$, the direction perpendicular to the slip direction on the slip plane is $\zeta^{(m)}$, and as to the spatial gradient of $\gamma^{(m)}$, the component in the direction parallel to the slip direction is an edge dislocation density component $\rho_{G, \text { edge }}^{(m)}$, and the component in the direction perpendicular to the slip direction is a screw dislocation density component $\rho_{G, s c r e w}^{(m)}$, then the norm of the GN dislocation density $\left\|\rho_{G}^{(m)}\right\|$ is represented by the following equation ${ }^{(10)}$ :

$$
\begin{aligned}
\left\|\rho_{G}^{(m)}\right\| & =\sqrt{\left(\rho_{G, \text { edge }}^{(m)}\right)^{2}+\left(\rho_{G, \text { screw }}^{(m)}\right)^{2}} \\
\rho_{G, \text { edge }}^{(m)} & =-\frac{1}{\tilde{b}} \frac{\partial \gamma^{(m)}}{\partial \xi^{(m)}} \\
\rho_{G, \text { screw }}^{(m)} & =\frac{1}{\tilde{b}} \frac{\partial \gamma^{(m)}}{\partial \zeta^{(m)}}
\end{aligned}
$$

\subsection{Deformation hysteresis and evaluation method}

The critical resolved shear stress $\theta^{(n)}$ in the slip system in a face-centered cubic crystal having been subjected to deformation is represented by the modified Bailey-Hirsch equation $^{(9)}$ :

$$
\theta^{(n)}=\theta_{0}(T)+\sum_{m=1}^{12} a \mu \tilde{b} \Omega^{(n m)} \sqrt{\rho_{s}^{(m)}}
$$

where, $\theta_{0}$ is a resistance value that does not depend on the deformation hysteresis against the moving dislocation, $a$ is a numerical value of about $0.1, \mu$ is an elastic shear modulus of crystal, and $\Omega^{(\mathrm{nm})}$ is an interactive matrix ${ }^{(9)} . \quad \Omega^{(\mathrm{nm})}$ indicates the strength of damage of the dislocations accumulated on the slip system imposed on the moving dislocation on another slip system, and can be represented by five kinds of parameters $R_{1}, R_{2}, R_{3}, R_{3}$, and $R_{4}$, the interactive strength between the dislocations on the same slip system being the reference unit. Here, isotropic hardening is supposed and so $R_{1}=R_{2}=R_{3}=R_{3}{ }^{\prime}=R_{4}=1.01$. This equation simplifies the multi-body interaction (thought to be extremely complex) that works between the moving dislocation and the stored dislocation into an abstract image in which the dislocation moves in the isotropic homogeneous field formed by the stored dislocations.

The hardening coefficient $h^{(n m)}$ of the strain of the material having been subjected to the deformation hysteresis is represented by the following equation:

$$
h^{(n m)}=\frac{1}{2} \frac{a c \mu \Omega^{(n m)}}{L^{(m)} \sqrt{\rho_{S}^{(m)}}}
$$

As a method of calculating the mean free path $L^{(n)}$ in equations (5) and (8), a dislocation density-dependent type model ${ }^{(11)(12)}$, in which the SS dislocation and the GN dislocation contribute, is used.

$$
L^{(n)}=\frac{c^{*}}{\sqrt{\sum_{m} \omega^{(n m)}\left(\rho_{S}^{(m)}+\left\|\rho_{G}^{(m)}\right\|\right)}}
$$

This model is based on an abstract image that the moving dislocation comes to a stop 
after having moved the distance $c^{*}$ times the average interval of the accumulated dislocations. Here, $c^{*}=15 . \omega^{(n m)}$ is a weighting matrix ${ }^{(11)}{ }^{(12)}$, which represents the interaction between the moving dislocation and the stored dislocation, and can be represented by six kinds of parameters $w_{0}, w_{1}, w_{2}, w_{3}, w_{3}$, and $w_{4}$. Here, considering that dislocations stored in the self-slip system and the common plane slip system do not contribute to the capture of the moving dislocations, it is assumed that $w_{0}=w_{1}=0$, and $w_{2}=$ $w_{3}=w_{3}{ }^{\prime}=w_{4}=1.0$.

Due to the contribution of the density of GN dislocations having size dependence on $L^{(n)}$, the GN dislocations concern the accumulation of the SS dislocations (refer to equation (5)), and the size effect occurs in the critical resolved shear stress and the strain hardening characteristics (refer to equations (7) and (8)).

\section{2:4 Increment analysis}

The present analysis consists of three parts, that is, the analysis of deformation (section 2.1 ), the calculation of the dislocation density (section 2.2), and the evaluation of strain hardening in the slip system (section 2.3).

At first, the constitutive equation of elastic deformation is determined from the elastic compliance and the initial crystal orientation, and the elastic deformation up to immediately before yield point in accordance with the boundary condition is analyzed. Next, an active slip system is selected by the Schmid law, a strain hardening coefficient is determined from the initial dislocation density, etc., the constitutive equation is modified, and the slip deformation is analyzed. Since strain hardening occurs in the slip system as deformation advances, it is necessary to dynamically modify the constitutive equation. To analyze the non-linear phenomenon, the increment analysis is performed by dividing time into steps each time the slip system starts to act and terminates. In other words, the SS dislocation density $\rho_{S}^{(m)}$ and the GN dislocation density norm $\left\|\rho_{G}^{(m)}\right\|$ serve as internal state variables for determining the constitutive equation in the next time step.

\section{Model}

The analysis model is symmetric type bicrystals as shown in Fig. 1, and the length $l$ of a side is $200 \mu \mathrm{m}$, and the thickness $t$ is $20 \mu \mathrm{m}$. The grain boundary plane is perpendicular to the model surface. Finite element division is carried out uniformly using eight-node hexahedron elements. The total number of elements is 6,400 .

GN dislocations dose not accumulate near the grain boundary after deformation when the bicrystal models subjected to deformation is represented by the following equation ${ }^{(13-15)}$ :

$$
\varepsilon_{y y}^{(1)}=\varepsilon_{y y}^{(2)}, \varepsilon_{z z}^{(1)}=\varepsilon_{z z}^{(2)}, \quad \varepsilon_{y z}^{(1)}=\varepsilon_{y z}^{(2)}
$$

where, $\varepsilon_{y y}^{(1)}, \varepsilon_{y y}^{(2)}$, etc., are strain components (the sum of the elastic strain component and the plastic strain component) produced when grains 1 and 2 deform independently of each other, and the superscripts denotes the crystal grain numbers.

The relationship between the crystal coordinate system constituted by the unit vectors

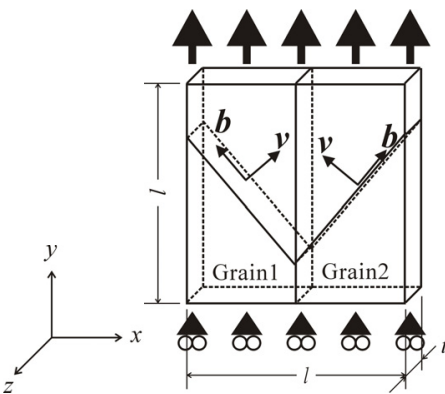

Fig. 1 Geometry and boundary condition for the models employed in this study

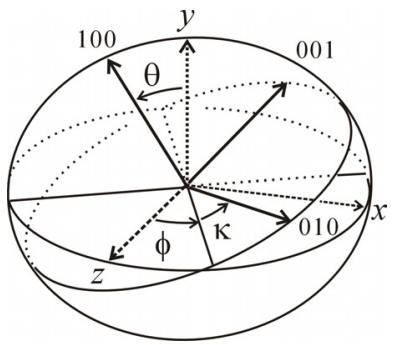

Fig. 2 Definition of Euler angles $\kappa, \theta$ and $\phi$ 
[100], [010], and [001] and the material coordinate system $(x, y, z)$ is defined by Euler angles $(\kappa, \theta, \phi)$ shown in Fig. 2.

There are several methods of defining Euler angles. By Euler angles, the coordinate transformation matrix between the material coordinate system and the crystal coordinate system is given by the following expression:

$$
\left(\begin{array}{l}
{[100]} \\
{[010]} \\
{[001]}
\end{array}\right)=\left(\begin{array}{ccc}
-\sin \theta \cos \phi & \cos \theta & \sin \theta \sin \phi \\
\cos \kappa \sin \phi+\cos \theta \sin \kappa \cos \phi & \sin \theta \sin \kappa & \cos \kappa \cos \phi-\cos \theta \sin \kappa \sin \phi \\
-\sin \kappa \sin \phi+\cos \theta \cos \kappa \cos \phi & \sin \theta \cos \kappa & -\sin \kappa \cos \phi-\cos \theta \cos \kappa \sin \phi
\end{array}\right)\left(\begin{array}{l}
x \\
y \\
z
\end{array}\right)
$$

It is assumed that the initial crystal orientation of grain 1 and 2 as shown in Table 1, the initial crystal orientation of grain 2 is one when grain 1 is rotated about the $y$-axis through 180 degrees. It is assumed that value of Schmid tensor in grain 1 and 2 under the tensile loading in the y-axis direction as shown in Table 2. In this case, since $v_{z}^{(9 t h)}$ and $b_{z}^{(9 t h)}$ are equal to 0 as to the primary slip system $(11 \overline{1})[101]$ in grains 1 and 2 , the components of Schmid tensor, $P_{33}^{(9 t h)}, P_{12}^{(9 t h)}, P_{23}^{(9 t h)}$ and $P_{31}^{(9 t h)}$ are also equal to 0 . Since the value of $P_{22}^{(9 t h)}$ and $P_{11}^{(9 t h)}$ for grains 1 and 2 are the same, the plastic strain increment $\dot{\varepsilon}_{y y}^{p}$ and $\dot{\varepsilon}_{x x}^{p}$ that occurs in grains 1 and 2 is equal, satisfying the compatible conditional expression (10), and incompatibility of shape change of the crystal grain due to the slip in the primary slip system does not occurs.

It is assumed that the combinations of the elastic compliances of the standard crystal orientation of Cu-9at.\% Al are $S_{11}=1.6793 \times 10^{-11}, S_{12}=-0.7130 \times 10^{-11}$ and $S_{44}=1.3055 \times$ $10^{-11}\left[\mathrm{~m}^{2} / \mathrm{N}\right]$, and so the elastic anisotropic ratio $2\left(S_{11}-S_{12}\right) / S_{44}$ is $3.66^{(16)}$.

The elastic compliance $S_{i j}^{(1)}, S_{i j}^{(2)}\left[\times 10^{-11} \mathrm{~m}^{2} / \mathrm{N}\right]$ of the material coordinate systems $(x, y$, $z$ ) are represented by the following equation:

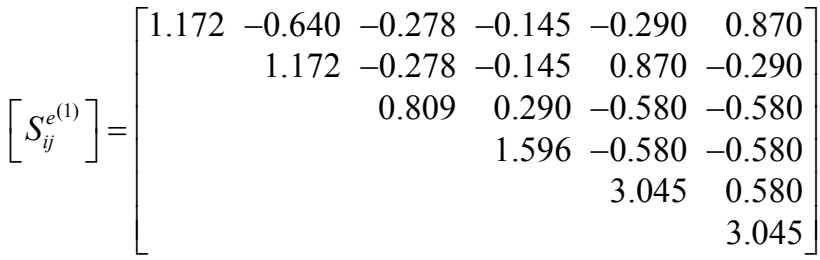

$$
\begin{aligned}
& {\left[S_{i j}^{e^{(2)}}\right]=\left[\begin{array}{rrrrrr}
1.172 & -0.640 & -0.278 & 0.145 & 0.290 & 0.870 \\
& 1.172 & -0.278 & 0.145 & -0.870 & -0.290 \\
& & 0.809 & -0.290 & 0.580 & -0.580 \\
& & & 1.596 & -0.580 & 0.580 \\
& & & & 3.045 & -0.580 \\
& & & & & 3.045
\end{array}\right]}
\end{aligned}
$$

where, the superscripts denotes the crystal grain numbers.

Table 1 Euler angle $(\kappa, \theta, \phi)$, components of normal direction and slip direction vector of the primary slip system

\begin{tabular}{|c|c|c|c|c|c|c|c|}
\hline Grain & Euler angle $(\kappa, \theta, \phi)$ & $b_{x}^{(9 t h)}$ & $b_{y}^{(9 t h)}$ & $b_{z}^{(9 t h)}$ & $v_{x}^{(9 t h)}$ & $v_{y}^{(9 t h)}$ & $v_{z}^{(9 t h)}$ \\
\hline 1 & $77.333,24.735,77.333$ & -0.7071 & 0.7071 & 0 & 0.7071 & 0.7071 & 0 \\
\hline 2 & $77.333,24.735,257.333$ & 0.7071 & 0.7071 & 0 & -0.7071 & 0.7071 & 0 \\
\hline
\end{tabular}

Table 2 Slip system number, definition of slip systems and Schmid factor

\begin{tabular}{|c|c|c|c|c|c|c|}
\hline Num. & 1 & 2 & 3 & 4 & 5 & 6 \\
\hline Slip system & $(111)[1 \overline{1} 0]$ & $(11)[01 \overline{1}]$ & $(111)[10 \overline{1}]$ & $(\overline{1} 11)[110]$ & $(\overline{1} 11)[01 \overline{1}]$ & $(\overline{1} 11)[110]$ \\
\hline Schmid Factor & 0.2875 & 0.1820 & 0.4694 & -0.2194 & -0.0527 & -0.1667 \\
\hline Num. & 7 & 8 & 9 & 10 & 11 & 12 \\
\hline Slip system & $(11 \overline{1})[1 \overline{1} 0]$ & $(11 \overline{1})[011]$ & $(11 \overline{1})[101]$ & $(1 \overline{1} 1)[110]$ & $(1 \overline{1} 1)[011]$ & $(1 \overline{1} 1)[10 \overline{1}]$ \\
\hline Schmid Factor & 0.2500 & 0.2500 & 0.5000 & 0.3180 & 0.1208 & 0.1973 \\
\hline
\end{tabular}


In this case, the value of elastic strain increment $\dot{\varepsilon}_{x x}^{e}, \dot{\varepsilon}_{y y}^{e}, \dot{\varepsilon}_{z z}^{e}, \dot{\varepsilon}_{z x}^{e}$ are equal under the tensile loading in the y-axis direction, however, since, signs of $\dot{\varepsilon}_{x y}^{e}$ and $\dot{\varepsilon}_{y z}^{e}$ are opposite (refer to equation (2)), the elastic strain incompatibility occur through the grain boundary plane (refer to equation (10)).

We assumed two type of initial dislocation density $\rho_{0}$, one is a uniform type and other is a non-uniform type. For the uniform type, It is assumed that the initial dislocation density $\rho_{0}$ is uniform, $1.0 \times 10^{9}\left[\mathrm{~m}^{-2}\right]$. For the non-uniform type, We gave the initial dislocation density $\rho_{0}=1.0 \times 10^{9}\left[\mathrm{~m}^{-2}\right]$ a standard deviation $S_{\rho 0}=1.0 \times 10^{8}\left[\mathrm{~m}^{-2}\right], 1.5 \times$ $10^{8}\left[\mathrm{~m}^{-2}\right]$ and $2.0 \times 10^{8}\left[\mathrm{~m}^{-2}\right]$.

The displacement in the $y$-axis direction at all the nodes on the bottom plane of the model is constrained, a uniform forced displacement in the y-axis direction is imposed on all the nodes on the top plane, and pulling is performed until the average tensile strain $\bar{\varepsilon}_{y y}$ becomes $1 \%$.

\section{Analysis Results and Consideration}

\section{4 - 1 Micro incompatibility and result of experimental study}

The surface observation image of $\mathrm{Cu}-9$ at.\%Al symmetric type bicrystals in the stage at which the average tensile strain $\bar{\varepsilon}_{y y}$ is $0.3 \%$ is shown in Fig. 3 . It is confirmed that the slip line in the primary slip systems (refer to Pri. line in Fig. 3) alternately appears on both sides of crystal grains and asymmetric deformation take place, even in the symmetric type bicrystals. At the same time, it is confirmed that secondary slip systems in the local area near the grain boundary is activated (refer to line Cri. and Conj. in Fig. 3), however, grain boundary plane does not work as dislocation source, that is, the so-called "micro incompatibility"(5) (6).

In this study, "micro incompatibility" accompanying tensile deformation in

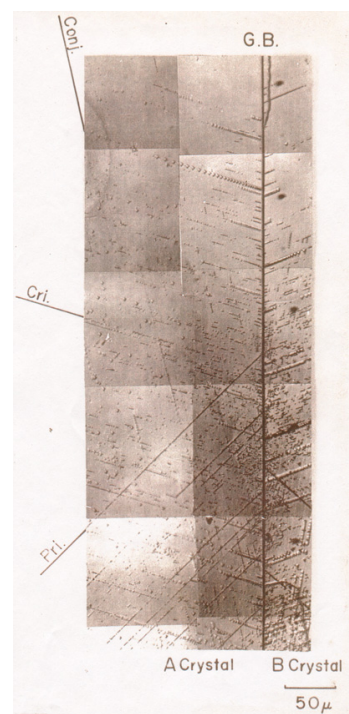

Fig. 3 Dislocation etch-pits configuration on slip lines observed in the symmetric type bicrystals when the average tensile strain $\bar{\varepsilon}_{y y}$ is $0.3 \%$. Cu-9at.\%Al symmetric type bicrystals depending on the elastic anisotropy, initial statically stored dislocations density, and a method of evaluating them, are described.

\section{4-2 Effect of elastic anisotropy}

The results of crystal plasticity analysis of tensile deformation in $\mathrm{Cu}-9 \mathrm{at} . \% \mathrm{Al}$ symmetric type bicrystals in the stage at which the average tensile strain $\bar{\varepsilon}_{y y}$ is $0.1 \%$ is shown in Fig. 4. Here, it is assumed that initial dislocation density $\rho_{0}$ is uniform, $1.0 \times 10^{9}$ $\left[\mathrm{m}^{-2}\right]$. For the plastic shear strain $\gamma^{(9 t h)}$ and the SS dislocation density $\rho_{S}^{(9 t h)}$ in the primary slip systems, the value from the maximum to the minimum is displayed, and for the GN dislocation density in the primary slip systems, the display range of edge dislocation density component $\rho_{G, \text { edge }}^{(9 t h)}$ and screw dislocation density component $\rho_{G, \text { screw }}^{(9 t)}$ are fixed to $-1.5 \times$ $10^{10}$ to $1.5 \times 10^{10}\left[\mathrm{~m}^{-2}\right]$, and the display range of the norm is fixed to 0 to $1.5 \times 10^{10}\left[\mathrm{~m}^{-2}\right]$. For the GN dislocation in the secondary slip systems, the display range of the edge dislocation density component $\rho_{G \text {,edge }}^{(3 r d)}$ and screw dislocation density component $\rho_{G, \text { screw }}^{(3 r d)}$ are fixed to $-3.0 \times 10^{9}$ to $3.0 \times 10^{9}\left[\mathrm{~m}^{-2}\right]$, and the display range of the norm is fixed to 0 to $3.0 \times 10^{9}\left[\mathrm{~m}^{-2}\right]$. 
It is conformed that asymmetric non-uniform deformation take place at the grain boundary, even in the symmetric type bicrystals, from distribution of plastic shear strain $\gamma^{(9 t h)}$ in the primary slip systems $(11 \overline{1})[101]$ (Fig.4 (a)). Here, the deformation models of the symmetric type bicrystals which having incompatibility of the elastic strain increment $\dot{\varepsilon}_{y z}^{e}$ (refer to equation (10)) are shown in Fig. $5^{(2)}$. In this case, the elastic incompatibility stress $\sigma_{y z}$ take place through the grain boundary plane, that is distributed asymmetrically and discontinuously in the grain boundary ${ }^{(4)}$, further, the shear stress component $\sigma_{x y}$ is distributed symmetrically in the grain boundary ${ }^{(4)}$.

Since, the elastic strain increment $\dot{\varepsilon}_{x y}^{e}$ (refer to equation (2), (12) and (13)) and shear stress component $\sigma_{x y}$ are distributed symmetrically in the grain boundary, restriction of deformation through the grain boundary plane also work symmetrically in the grain boundary. Since, $v_{z}^{(9 t h)}=b_{z}^{(9 t h)}=0$, the plastic share strain gradient occurs in the slip direction, and therefore, the GN dislocation structure that is composed of edge dislocation density component, is formed (Fig. 4 (c)). Then, the GN dislocation in which edge and screw dislocation density components are mixed, is accumulated at the grain boundary (Figs 4(b)-(d)), further, the strain hardening is develop with accumulation of SS dislocation at the grain boundary (Figs 4(e)).

Since, the spatial arrangement of the primary slip system and the secondary slip system depends on the crystal orientation, the activation of secondary slip systems is necessary for the deformation in the local area near the grain boundary. The area in which the GN dislocation have accumulated in the secondary slip systems (Figs. 4(f)-(h)), in this case, that is displayed in the grain2 only. However, we have confirmed that the GN dislocation in the secondary slip systems accumulated in the grain 1 from the back of model. The results in which GN dislocation in the secondary slip systems have accumulated asymmetrically and discontinuously, are conformed that the shear stress $\sigma_{y z}$ have distributed asymmetrically and discontinuously in the grain boundary, at the same time, the grain boundary plane dose not work as dislocation source.

The results of crystal plasticity analysis of tensile deformation in $\mathrm{Cu}-9 \mathrm{at} . \% \mathrm{Al}$ symmetric type bicrystals in the stage at which the average tensile strain $\bar{\varepsilon}_{y y}$ is $1 \%$ is shown in Fig. 6. Here, for the plastic shear strain $\gamma^{(9 t h)}$ and the SS dislocation density $\rho_{S}^{(9 t h)}$ in the primary slip systems, the value from the maximum to the minimum is displayed, and for the GN dislocation density in the primary slip systems, the display range is fixed for

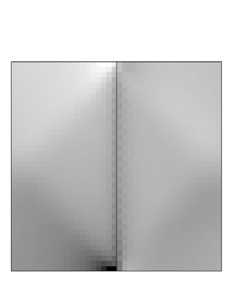

(a) $\gamma^{(9 t h)}$

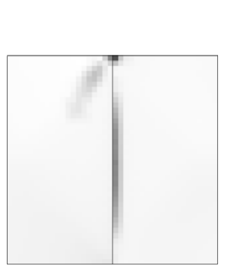

(e) $\rho_{S}^{(9 t h)}$
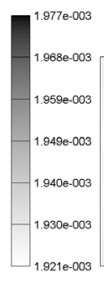

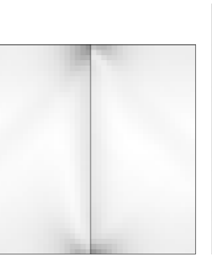

(b) $\left\|\rho_{G}^{(9 t h)}\right\|$

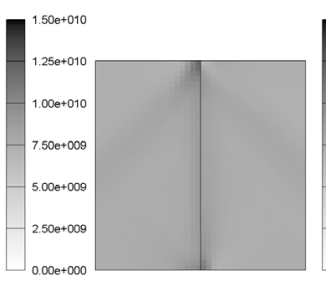

(c) $\rho_{G, e d g e}^{(9 t h)}$

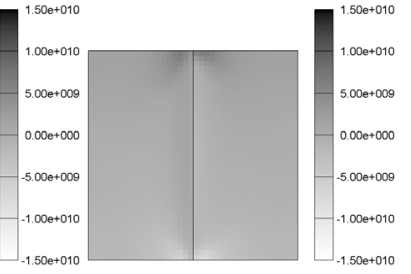

(d) $\rho_{G, \text { screw }}^{(9 t h)}$

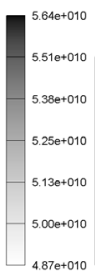

$5.00+0+010$

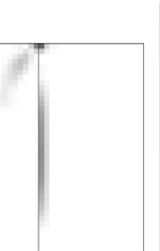

(f) $\left\|\rho_{G}^{(3 r d)}\right\|$

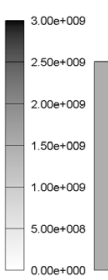

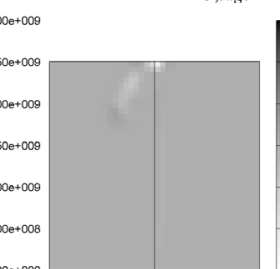

(g) $\rho_{G, e d g e}^{(3 r d)}$

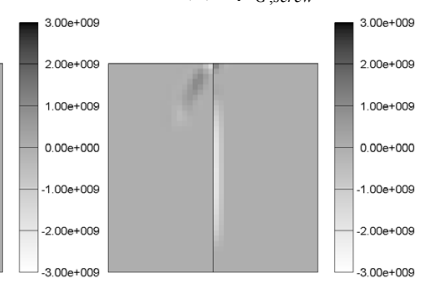

(h) $\rho_{G, s c r e w}^{(3 r d)}$

Fig. 4 (a): Distribution of plastic shear strain $\gamma^{(9 t h)}$, density distribution of (b): norm, (c): edge and (d): screw components of geometrically necessary dislocations and (e): statistically stored dislocations on the primary slip system $(11 \overline{1})[101]$, density distribution of (f): norm, (g): edge and (h): screw components of geometrically necessary dislocations on the secondary slip systems (111) $[10 \overline{1}]$ when the average tensile strain $\bar{\varepsilon}_{v v}$ is $0.1 \%$, respectively. Unit of dislocation density is $\mathbf{m}^{-2}$. 
comparison.

Under the this analysis condition, the incompatibility of plastic strain component through the grain boundary plane does not take place, at the same time, the restriction of shape change of crystal grain by free slip in the primary slip systems also does not occur (13)-(15). Therefore, the deformation constraints in which works interactively between crystal grains through the grain boundary due to the effects of the elastic anisotropy, influence on the results of analysis in the later stage of plastic deformation. The strain hardening developments strongly depend on the GN dislocation structure in which accumulated near the grain boundary in the early stage of deformation, and the GN dislocation structure and the non-uniform deformation field develops remarkably. From the result described above, it has been found that the activation of secondary slip systems in the local area near the grain boundary accompanying tensile deformation of $\mathrm{Cu}-9 \mathrm{at} . \% \mathrm{Al}$ symmetric type bicrystals strongly depend on the elastic anisotropy of materials.

\section{4 - 3 Effects of heterogeneity of initial dislocation density}

The results of surface observation accompanying tensile deformation of $\mathrm{Cu}-9 \mathrm{at} . \% \mathrm{Al}$ symmetric type bicrystals, the asymmetric distribution of the slip line in the primary slip systems and the microscopic asymmetric deformation take place in the grain boundary. Only in the effects of the elastic anisotropy, the asymmetric dynamical phenomena are not described completely. Here, it is discussed from the viewpoint of the influence of the heterogeneity in which inheres in the actual material. The various factors are supposed for the heterogeneity in which inheres in the actual material, however, here, particularly investigate the influence in which gave the initial dislocation density $\rho_{0}=1.0 \times 10^{9}\left[\mathrm{~m}^{-2}\right] \mathrm{a}$

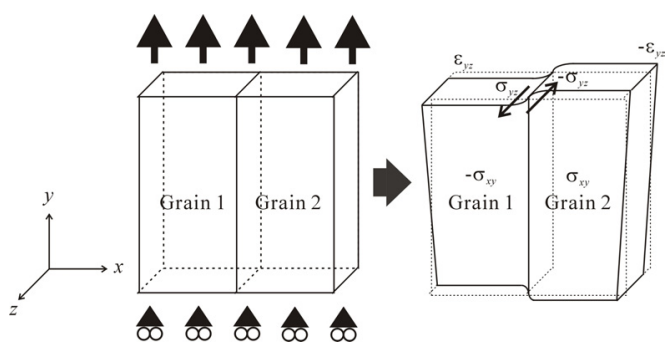

Fig. 5 Schematic illustration of deformation of model showing how stresses $\pm \sigma_{x v}, \pm \sigma_{v z}$ arise from incompatibility of components of elastic strains $\dot{\varepsilon}_{y z}^{e} \quad$ under the tensile loading

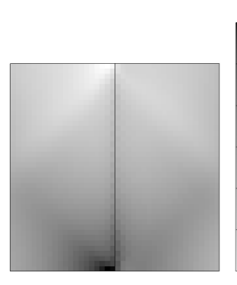

(a) $\gamma^{(9 t h)}$

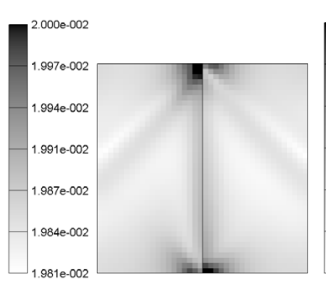

(b) $\left\|\rho_{G}^{(9 t h)}\right\|$

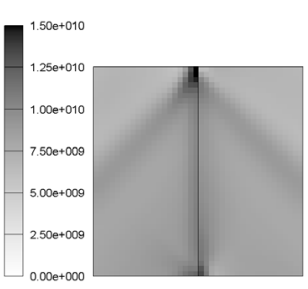

(c) $\rho_{G, e d g e}^{(9 t h)}$

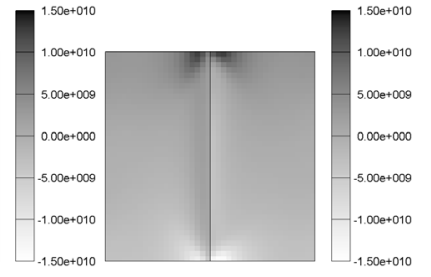

(d) $\rho_{G, s c r e w}^{(9 t h)}$

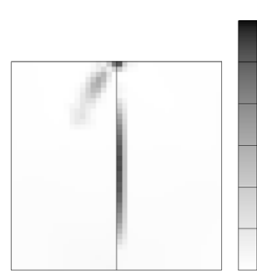

(e) $\rho_{S}^{(9 t h)}$

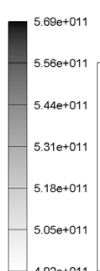

$5.05+011$

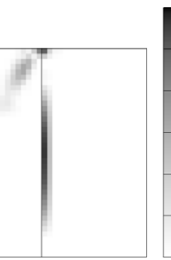

(f) $\left\|\rho_{G}^{(3 r d)}\right\|$

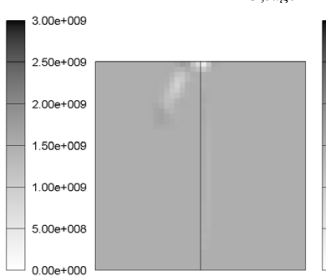

(g) $\rho_{G, e d g e}^{(3 r d)}$

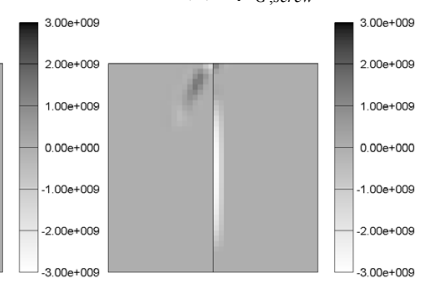

(h) $\rho_{G, s c r e w}^{(3 r d)}$

Fig. 6 (a): Distribution of plastic shear strain $\gamma^{(9 t h)}$, density distribution of (b): norm, (c): edge and (d): screw components of geometrically necessary dislocations and (e): statistically stored dislocations on the primary slip system $(11 \overline{1})[101]$, density distribution of (f): norm, (g): edge and (h): screw components of geometrically necessary dislocations on the secondary slip systems $(111)[10 \overline{1}]$ when the average tensile strain $\bar{\varepsilon}_{y y}$ is $1.0 \%$, respectively. Unit of dislocation density is $\mathbf{~ m}^{-2}$. 
standard deviation $S_{\rho 0}=1.0 \times 10^{8}\left[\mathrm{~m}^{-2}\right], 1.5 \times 10^{8}\left[\mathrm{~m}^{-2}\right]$ and $2.0 \times 10^{8}\left[\mathrm{~m}^{-2}\right]$.

Distribution of the GN dislocation density norm accompanying tensile deformation in $\mathrm{Cu}-9$ at.\%Al symmetric type bicrystals in the stage at which the average tensile strain $\bar{\varepsilon}_{y y}$ is $1 \%$ is shown in Fig. 7. Here, the display range of the GN dislocation density norm is fixed for comparison with Fig. 4 and Fig. 6. By the influence of the heterogeneity of the initial dislocation density, the GN dislocations accumulate in a high density in a band-like area that has developed in the direction perpendicular to the slip direction in the primary slip systems. Since the asymmetric GN dislocation structures are distributed in the grain boundary, the result of analysis are accordance with the experimental results of observation in $\mathrm{Cu}-9 \mathrm{at} . \% \mathrm{Al}$ symmetric type bicrystals, and the GN dislocation density in the secondary slip systems near the grain boundary are also distributed non-uniformly.

\section{$4 \cdot 4$ Evaluation of micro incompatibility by disclination type deformation field}

In the case of the crystal grain have heterogeneity of initial dislocation density, the microscopic non-uniform deformation is understood to have two stages, as shown in Figs.

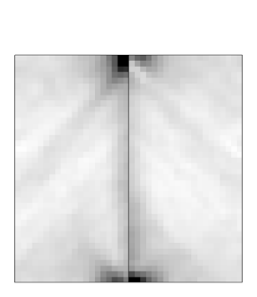

(a): $S_{\rho 0}=1.0 \times 10^{8}\left[\mathrm{~m}^{-2}\right]$

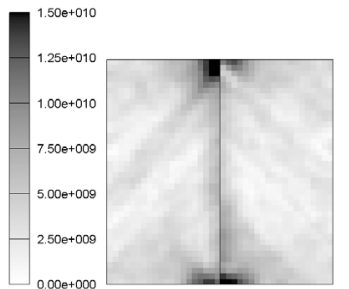

(b): $S_{\rho 0}=1.5 \times 10^{8}\left[\mathrm{~m}^{-2}\right]$

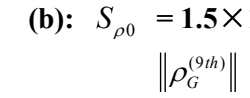

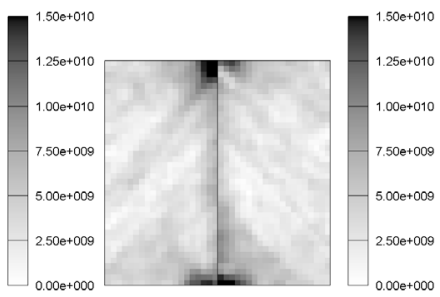

(c): $S_{\rho 0}=2.0 \times 10^{8}\left[\mathrm{~m}^{-2}\right]$
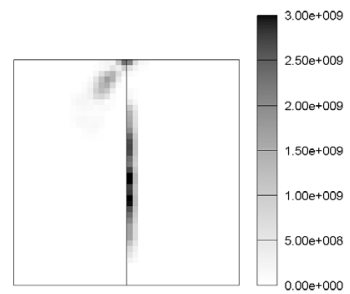

(d): $S_{\rho 0}=1.0 \times 10^{8}\left[\mathrm{~m}^{-2}\right]$

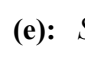

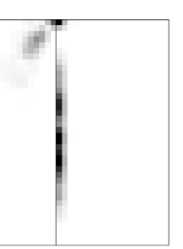

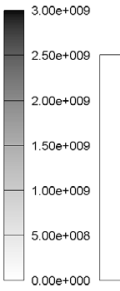

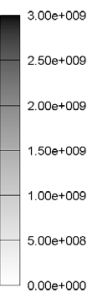

$0.000+000$

Fig. 7 Density distribution of norm of geometrically necessary dislocation on the (a)-(c) : primary $(11 \overline{1})[101]$ and (d)-(f): secondary slip systems (111)[10 $\overline{1}]$ when the average tensile strain $\bar{\varepsilon}_{v v}$ is $1 \%$, respectively. Standard deviation of initial statically stored dislocation $S_{\rho 0}$ is (a),(d): $1.0 \times 10^{8}$ $\left[\mathrm{m}^{-2}\right],(\mathrm{b}),(\mathrm{e}): 1.5 \times 10^{8}\left[\mathrm{~m}^{-2}\right]$ and $(\mathrm{c}),(\mathrm{f}): 2.0 \times 10^{8}\left[\mathrm{~m}^{-2}\right]$ respectively.

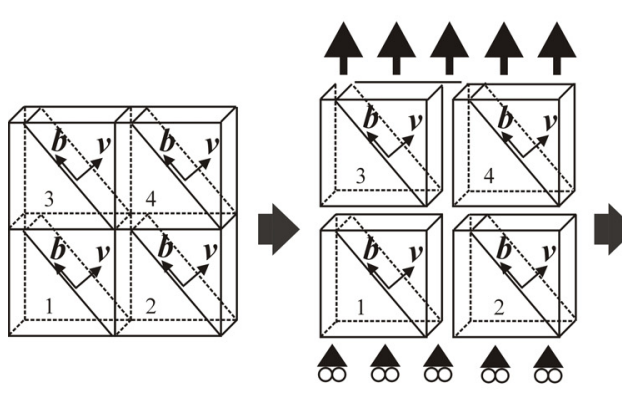

(a)

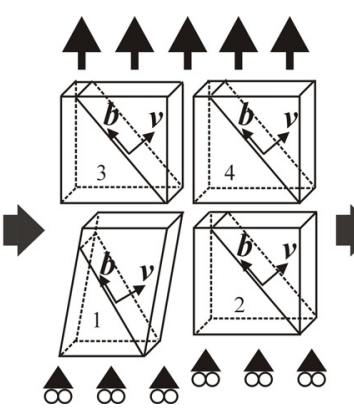

(b)

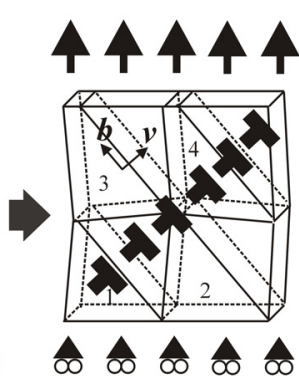

(c)

Fig. 8 Schematic illustration of (a): initial condition local area, (b): imaginary slip deformation without binding interaction, (c): imaginary wedge disclination type deformation with binding interaction. 


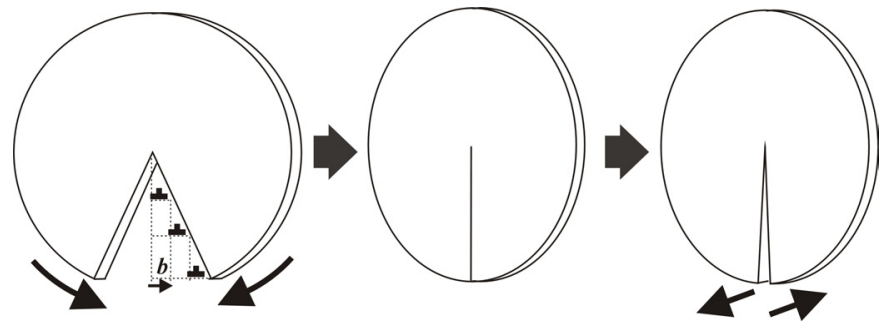

(a)

(c)

Fig. 9 Schematic illustration of (a): Initial condition with dislocation, (b): wedge and (c): twist disclination type deformation.

8(a)-(b) and (b)-(c). Figures 8(a)-(b) shows the activation and free deformation of microscopic local areal that are caused by slip on the primary slip system, in this stage, the shape change of the microscopic local area occur depend on the initial crystal orientation and plastic shear strain $\gamma^{(9 t h)}$. Figures 8(b)-(c) shows the interaction of microscopic local areas in order to satisfy the continuity of displacement and the resulting non-uniform deformations, in this stage, the displacement field is similar to that for disclination type deformation field in which core is the intersection of microscopic local areas 1, 2, 3 and 4.

The disclination type deformation field is formed by relative rotation from the defective situation to normality, as shown in Figs.9, in the case of the deformation field, as shown in Figs. 9(a)-(b), that is, so called "Wedge disclination", and in the case of twist deformation, as shown in Fig9(c), that is, so called "Twist disclination". Wedge disclination defects spread depending on the distance from their core, and their direction is similar to the line of the edge dislocations when the direction of the disclination core is perpendicular to the direction of the Burgers vector, as shown in Fig. 9(a). Since the line of edge dislocation is geometrically required to exist on the primary slip plane, the pattern of GN edge dislocation develops in the direction perpendicular to the slip direction in the primary slip systems. Since the GN edge dislocation line form the inner stress field, in the circumference, the primary stress axis deviates from the load direction, and an increase/decrease in the critical resolved shear stress in the primary slip system and the secondary slip system, at the same time, the secondary slip system is activated discontinuously in the microscopic local area (Figs. 7(d)-(f)). Since the strain hardening due to the dislocation interaction also occurs discontinuously in the microscopic local area, the microscopic asymmetric deformation develops with the advancement of deformation. Since many disclination cores are existent in the heterogynous material, the microscopic GN dislocation structures are formed in the whole area in the crystal grain.

\section{Conclusion}

The relationship between the mechanism of "micro-incompatibility" at the grain boundary accompanying tensile deformation of $\mathrm{Cu}-9 \mathrm{at} . \% \mathrm{Al}$ symmetric type bicrystals and the elastic anisotropy, and influence of the heterogeneity of initial dislocation density imposed on the after deformation stage, were obtained by performing crystal plasticity analysis of tensile deformation of $\mathrm{Cu}-9$ at.\% $\mathrm{Al}$ symmetric type bicrystals. The results are as follows.

(1) The activation of secondary slip systems in the local area near the grain boundary accompanying tensile deformation of $\mathrm{Cu}-9 \mathrm{at} . \% \mathrm{Al}$ symmetric type bicrystals is caused by the elastic incompatibility stress due to the elastic anisotropy.

(2) The asymmetric distribution of the slip line in the primary slip systems in the grain boundary accompanying tensile deformation of $\mathrm{Cu}-9 \mathrm{at} . \% \mathrm{Al}$ symmetric type bicrystals, that is ,the so called "micro-incompatibility", is caused by the heterogeneity in which inheres in the actual material. 
(3) The heterogeneity in which inheres in the actual material form the GN dislocation structure and the inner stress field, in the circumference, an increase/decrease in the critical resolved shear stress in the primary slip system and the secondary slip system, at the same time, non-uniformity of deformation develops abruptly.

(4) Non-uniform deformation field in which is caused by the heterogeneity, is similar to that for disclination type deformation field, microscopic non-uniform deformation and microscopic GN dislocation structure formation are possible for relative and evaluation.

\section{References}

(1) Hirth, J. P., The influence of grain boundaries on mechanical properties, Metall. Trans., Vol. 3 (1972) pp. 3047-3067.

(2) Hook, R. E. and Hirth, J. P., The Deformation Behavior of Isoaxial Bicrystals of Fe-3\% Si, Acta Metall., Vol. 15 (1967) pp. 535-551.

(3) Livingston, J. D. and Chalmers, B., Multi Slip in Bicrystal Deformation, Acta Metall., Vol. 5, No. 6 (1957) pp. 322-327.

(4) Ohashi T., et al., Elastic Incompatibility Stress in Bicrystal Body, J. Japan Inst. Metals, Vol. 44, No. 8 (1980) pp. 876-883.

(5) Miura S., et al., Study on The Deformation Mechanism of Cu-9at.\% Al Alloy Bicrystals using an Etch Pitting Technique, Suiyo Kwai-shi, Vol. 19, No. 9 (1982) pp. 673-685.

(6) Hashimoto S. and Miura S., Dislocation Arrays and the Bauschinger Effects in Copper 9at.\% Aluminum Bicrystals, Mem. Fac. Eng. Kyoto Univ., Vol. 44, No. 1 (1986) pp. 1-29.

(7) Hill R., Generalized Constitutive Relations for Incremental Deformation of Metal Crystals by Multislip, J. Mech. Phys. Sol., Vol. 14 (1966), pp. 95-102.

(8) Ashby M. F., The Deformation of Plastically Non- homogeneous Alloys, Phil. Mag., Vol. 21 (1970) pp. 399-424.

(9) Ohashi T., Numerical Modeling of Plastic Multislip in Metal Crystals of F.C.C. Type, Phil. Mag. A, Vol. 70, No. 5 (1994) pp. 793-803.

(10) Ohashi T., Finite-Element Analysis of Plastic Slip and Evolution of Geometrically Necessary Dislocations in F.C.C. Crystals, Phil. Mag. Lett. Vol. 75, No. 2 (1997) pp. 51-57.

(11) Ohashi T., A New Model of Scale Dependent Crystal Plasticity Analysis, IUTAM Symposium on Mesoscopic Dynamics of Fracture Process and Materials Strength (2004) pp. 97-106.

(12) Ohashi T., Prediction of Macroscopic Mechanical Response of Metallic Materials Through Evaluation of Deformation Processes in Microstructure, The Iron and Steel Institute of Japan, 180 181 Nishiyama Anniversary Technique Lecture (in Japanese) (2004), pp. 73-95.

(13) Kondou R. and Ohashi T., Crystal Plasticity Analysis of Non-uniform Deformation in Symmetric Type Bicrystals under Tensile Load and Formation of Geometrically Necessary Dislocation Bands, Journal of Solid Mechanics and Materials Engineering, Vol. 1, No. 1 (2007) pp.114-125.

(14) Kondou R. and Ohashi T., Relationship between Formation of Geometrically Necessary Dislocations and Local Strain hardening of Slip Systems in Symmetric type Bi-Crystals under Tensile Loading, Journal of Solid Mechanics and Materials Engineering, Vol. 1, No. 6 (2007) pp. 755-766.

(15) Kondou R. and Ohashi T., Grain Boundary Accumulation of Geometrically Necessary Dislocation and Asymmetric Deformation in Compatible Bicrystals with Tilt Angle Grain Boundary under Tensile Loading, International Journal of The Japan Society of Mechanical Engineers, A, Vol. 49, No. 4 (2006) pp.581-588

(16) Simmons, G. and Wang, H., Single Crystal Elastic Constants and Calculated Aggregate Properties, Second Edition (1971) 24, The M.I.T. press. 